\title{
Psychological state, quality of life, and body composition in postmenopausal women with osteoporosis in Lithuania
}

\author{
Lina Lasaite • Aurelija Krasauskiene
}

Received: 10 September 2009 / Accepted: 4 November 2009/Published online: 1 December 2009

(C) The Author(s) 2009. This article is published with open access at Springerlink.com

\begin{abstract}
Summary The objective of this study was to determine body composition, physical activity, and psychological state in postmenopausal women with osteoporosis. Fat mass, lean mass, water mass, and basal metabolic rate are lower, self-reported physical activity and risk factors of fractures are higher, and cognitive functions were worse in osteoporotic patients than in controls. Significant correlations were found between physical activity and emotional state parameters.

Introduction This study aims to determine peculiarities of body composition, physical activity, risk factors predicting fractures, psychological state and quality of life, and possible relations between them in postmenopausal women with osteoporosis in Lithuania.

Methods Thirty-one postmenopausal women with osteoporosis and 29 healthy age- and sex-matched controls were included in the study. Profile of Mood State and Hospital Anxiety and Depression Scale were used for the assessment of emotional state. Trail Making Test and Digit Symbol Test of Wechsler Adult Intelligence Scale were used to evaluate cognitive functioning. Quality of life was evaluated using the World Health Organization Brief Quality of Life Questionnaire. Risk of fractures was assessed by the Risk Factors Predicting Questionnaire.

Results Fat mass $(22.4 \pm 4.7$ vs. $40.6 \pm 14.2 \mathrm{~kg}, p<0.001)$, lean mass $(37.3 \pm 6.0$ vs. $48.1 \pm 7.6 \mathrm{~kg}, p<0.001)$, water mass $(31.6 \pm 2.9$ vs. $38.3 \pm 5.3 \mathrm{~kg}, p<0.001)$, and basal metabolic rate $(1,253 \pm 132$ vs. $1,456 \pm 126 \mathrm{kcal}, p<0.001)$ were lower in osteoporotic patients than in controls. Self-
\end{abstract}

L. Lasaite $(\triangle) \cdot$ A. Krasauskiene

Institute of Endocrinology, Kaunas University of Medicine,

Eiveniu 2,

50009 Kaunas, Lithuania

e-mail: linlasla@yahoo.com reported physical activity $(2.35 \pm 0.6$ vs. $1.69 \pm 0.5, p<$ $0.001)$ and risk factors of fractures $(5.9 \pm 2.1$ vs. $2.6 \pm 2.4$, $p<0.001)$ were higher in women with osteoporosis than in healthy age- and sex-matched controls $(2.35 \pm 0.6$ vs. $69 \pm 0.5, p<0.001)$. Trail making $\mathrm{A}$ and $\mathrm{B}$ scores were higher in patients than in age- and sex-matched controls (55.8 \pm 19.9 vs. $45.1 \pm 19.9, p=0.07$ and $118.2 \pm 34.6$ vs. $92.8 \pm 48.7, p=0.006)$. Some significant correlations were detected between physical activity and emotional state and quality of life parameters.

Conclusion In postmenopausal women with osteoporosis, fat body mass, lean body mass, water body mass, basal metabolic rate, and waist-to-hip ratio are lower, physical activity and risk of fractures are higher, and cognitive functions are worse than in age- and sex-matched controls. Some psychological peculiarities could be related to physical activity in women with osteoporosis.

Keywords Body composition - Cognitive functions . Emotional state · Osteoporosis · Postmenopausal women . Quality of life

\section{Introduction}

As the population of developed countries age, osteoporosis becomes a significant public health problem [1]. Osteoporosis is a systemic disease in which bone density is reduced, leading to the weakening of the skeleton and increased vulnerability to fractures [2]. It is a widespread disease affecting about 75 million people, mostly postmenopausal women. It is called "the silent disease" since there are very few associated symptoms; osteoporotic fractures are the chief clinical feature with an enormous burden on healthrelated quality of life and mortality [3]. 
At the beginning of the osteoporosis studies in Lithuania in 1991-1993, the rate of characteristic osteoporotic fractures in Kaunas region (580,000 inhabitants; 170,000 males and 350,500 females), Lithuania, was found to be $1.60-1.65 \%$ in males over 65 years old and $7.52-10.56 \%$ in females over 65 years old [4]. Bone mineral density (BMD) measurements for healthy Lithuanian females are not significantly different from the normative Caucasian female reference data both in terms of average values and degree of spread. The Lithuanian women show the expected gradual reduction of BMD with age, after peaking at 20-39. The BMD of Lithuanian women tends to mirror rates of loss established for US/European subjects [5].

Increased likelihood of fracture has often been expressed as relative risk or risk ratio, defined as the ratio of fracture occurrence among individuals with, versus without, a given risk factor. However, relative risk alone is inadequate to express actual outcome frequency. More informative than relative risk is an approach that estimates the absolute risk of an individual with a given constellation of factors [age, sex, body mass index (BMI), prior fracture, parental hip fracture, bone mass density, smoking, and so on]. Data from population-based studies of osteoporotic fractures subjects were used to produce multivariable osteoporotic fractures prediction questionnaire models [6].

Osteoporosis can reduce mobility and may be painful, which can limit everyday activities, can lead to increasing isolation, which has a negative impact upon self-esteem, causes depression, and affects emotional state and quality of life [7, 8]. Quality of life plays an increasingly important role in the evaluation of the impact of osteoporosis. Quality of life may be impaired through the physical disability and psychological and social handicaps resulting from fractures [8].

Depressive symptoms are associated with increased risk of osteoporosis and new fractures even after controlling for other predictive factors [9]. Dual diagnosis of depression and osteoporosis may also mean worse health outcomes [10]. Perceived risk of osteoporosis may lead to decreased physical activity and hence actually decrease physical activity, change body composition, and increase the health-associated risk [11].

Osteoporosis can also cause both social consequences and psychological difficulties for patients with this disease: loss of social roles, failure in social reciprocity, social isolation, loneliness, depression, anxiety, reduced selfworth, and hopelessness. However, the causal direction of relationships between these factors and poor health is unclear. Since 1990, when the coexistence of osteoporosis and depression was first noted in observational studies [1], it is not clear whether osteoporosis can be seen as the causal factor of depression or depression as the outcome of osteoporosis [12]. Although causality is not clear, in addition to low BMI, role limitations due to poor emotional status and low physical functioning are related to low bone mineral content in postmenopausal women [13].

In the study of quality of life in Lithuanian women with osteoporosis, no significant differences were assessed between women with subclinical vertebral fractures and healthy women. Women with one subclinical vertebral fracture showed significantly worse results in pain, social function, and general health perception domains of quality of life [14].

Therefore, a lot is unclear in the relationship between physical status, psychological state, and quality of life, and a lot of data of research studies are quite controversial. But the results imply that, when we are concerned about osteoporosis, we should pay attention not only to the physical function but also to the psychological state of patients [13].

The objective of the study was to determine peculiarities of body composition, physical activity, osteoporotic fracture risk prediction, psychological state and quality of life, and possible relations between them in postmenopausal women with osteoporosis.

\section{Patients and methods}

All procedures were carried out with the adequate understanding and written consent of the subjects.

Thirty-one postmenopausal women (average age 69.3 \pm 8.2 years) with osteoporosis (members of a disease-related club) and 29 healthy age- and sex-matched controls (average age 66.2 \pm 6.3 years) were included in the study. The inclusion of subjects was random.

Height was measured by stadiometer of international standard "Holtain Limited, Crymic Dyteg" (UK) and weight by electronic weights "Soehnle" (UK). Body composition was evaluated by means of a body composition monitoring unit Bodystat 1500 (UK).

Profile of Mood State (POMS) [15] and Hospital Anxiety and Depression Scale (HAD) [16] were used for the assessment of emotional state. The POMS questionnaire is a sensitive measure of mood. It measures POMS global score and several subscales: tension-anxiety, depressiondejection, anger-hostility, vigor-activity, fatigue-inertia, and confusion-bewilderment. A higher score represents a higher level of certain mood aspect.

Cognitive functioning (evaluation of psychomotor speed) was detected by use of Trail Making Test [17] and Digit Symbol Test of the Wechsler Adult Intelligence Scale [18]. Trail Making Test is a timed test in which the subject connects an altering sequence of numbers (trail making A) or numbers and letters (trail making B) in ascending order. The score on the test, which is based on the time required to 
complete the sequence, is a measure of executive function, attention, and visual scanning abilities. Digit Symbol Test is a test in which the subject repeats sequences of numbers in a given order. The score of the test is a number or repeated number sequences in forward order, backward order, and global score (sum of both of them). The test shows attention abilities and psychomotor speed.

Quality of life was evaluated by means of the World Health Organization Brief Quality of Life Questionnaire [19]. It evaluates general satisfaction with health-related quality of life of the subject.

Osteoporotic fracture risk was evaluated by Factors Predicting Osteoporotic Fractures Questionnaire [6], which evaluates risk of fractures according to bone mineral density, age, sex, smoking or not, and physical state of the subject. Physical activity was self-reported by the participants of the study and was evaluated as low, middle, or high.

Descriptive values are given as mean \pm standard deviation. The differences between data of the researched group and the control group were compared using MannWhitney $U$ test. Correlations were found by means of Pearson test. In all calculations, we considered $p$ values $<0.05$ as significant and $p$ values $<0.09$ as tendency.

\section{Results}

Table 1 shows the comparison of anthropometric and body composition data in osteoporotic postmenopausal women and sex- and age-matched controls. Body mass index and waist-to-hip ratio of women with osteoporosis were significantly lower than in the controls. Also, all evaluated body composition parameters were significantly lower in osteoporotic patients than in the controls: fat body mass, lean body mass, water body mass, and basal metabolic rate. Physical activity was self-reported by the participants of the study themselves and was significantly higher in postmenopausal women with osteoporosis than in healthy age- and sex-matched controls. Risk of fractures was significantly higher in osteoporotic postmenopausal women than in healthy controls.

In Table 2, comparison of psychological data and quality of life between osteoporotic patients and healthy women is presented. Significant differences were observed in cognitive functioning but not in emotional state and quality of life. Trail making A and B scores were higher in patients with osteoporosis than in age- and sex-matched controls, showing worse cognitive functioning of osteoporotic women.

Some significant correlations were detected (Table 3) between physical activity and emotional and quality of life parameters. Depression and anxiety correlated negatively and quality of life correlated positively with physical activity.

\section{Discussion}

The results of our study confirm that not only BMI, weight, and fat mass are lower in women with osteoporosis than in age- and sex-matched controls but also that lean body mass, water body mass, and waist-to-hip ratio are lower and selfreported physical activity is higher.

It is well known that bone mineral density increases with weight and body fat mass, and obesity has a protective effect against osteoporosis [20, 21]. Some studies indicate that both fat mass and lean mass affect bone density with different physiological and pathological conditions modulating this relationship [22]. Our study confirms that not only weight, BMI, and fat body mass but also lean body mass, water body mass, basal metabolic rate, and waist-tohip ratio in postmenopausal women with osteoporosis are significantly lower than in age- and sex-matched controls.

The protective effect of higher body weight is explained by a combination of hormonal (peripheral aromatization of
Table 1 Anthropometric, body composition, physical activity, and osteoporotic fracture risk data in postmenopausal women with osteoporosis in comparison to age- and sex-matched controls

$N S$ not significant

\begin{tabular}{lccl}
\hline & $\begin{array}{c}\text { Postmenopausal women } \\
\text { with osteoporosis }(n=31)\end{array}$ & $\begin{array}{c}\text { Age- and sex-matched } \\
\text { controls }(n=29)\end{array}$ & $\begin{array}{l}p \text { value (Mann- } \\
\text { Whitney test) }\end{array}$ \\
\hline Age (years) & $69.3 \pm 8.2$ & $66.2 \pm 6.3$ & $\mathrm{NS}$ \\
Height $(\mathrm{cm})$ & $158.5 \pm 6.5$ & $159.2 \pm 4.6$ & $\mathrm{NS}$ \\
Weight $(\mathrm{kg})$ & $59.7 \pm 9.1$ & $86.5 \pm 19.2$ & $<0.001$ \\
Body mass index $\left(\mathrm{kg} / \mathrm{m}^{2}\right)$ & $23.7 \pm 3.1$ & $34.2 \pm 7.5$ & $<0.001$ \\
Fat body mass $(\mathrm{kg})$ & $22.4 \pm 4.7$ & $40.6 \pm 14.2$ & $<0.001$ \\
Lean body mass $(\mathrm{kg})$ & $37.3 \pm 6.0$ & $48.1 \pm 7.6$ & $<0.001$ \\
Water body mass $(\mathrm{kg})$ & $31.6 \pm 2.9$ & $38.3 \pm 5.3$ & $<0.001$ \\
Basal metabolic rate $(\mathrm{kcal})$ & $1253 \pm 132$ & $1456 \pm 126$ & $<0.001$ \\
Waist-to-hip ratio & $0.82 \pm 0.45$ & $0.86 \pm 0.64$ & 0.01 \\
Physical activity & $2.35 \pm 0.6$ & $1.69 \pm 0.5$ & $<0.001$ \\
Risk of osteoporotic fractures & $5.9 \pm 2.1$ & $2.6 \pm 2.4$ & $<0.001$ \\
\hline
\end{tabular}


Table 2 Psychological and quality of life data in postmenopausal women with osteoporosis in comparison to age- and sex-matched controls

NS not significant

${ }^{a}$ The higher score denotes worse cognitive functioning

${ }^{\mathrm{b}}$ The higher score denotes better cognitive functioning

${ }^{\mathrm{c}}$ The higher score denotes worse emotional state

${ }^{d}$ The higher score denotes better emotional state

${ }^{\mathrm{e}}$ The higher score denotes better quality of life
Postmenopausal women

with osteoporosis $(n=31)$
Age- and sex-matched $p$ value (Manncontrols $(n=29) \quad$ Whitney test $)$

\begin{tabular}{|c|c|c|c|}
\hline \multicolumn{4}{|l|}{ Cognitive functioning: } \\
\hline Trail making $\mathrm{A}^{\mathrm{a}}$ & $55.8 \pm 19.9$ & $45.1 \pm 19.9$ & 0.07 \\
\hline Trail making $\mathrm{B}^{\mathrm{a}}$ & $118.2 \pm 34.6$ & $92.8 \pm 48.7$ & 0.006 \\
\hline Digit symbol, global score ${ }^{b}$ & $11.0 \pm 2.3$ & $10.2 \pm 3.1$ & NS \\
\hline \multicolumn{4}{|l|}{ Emotional state: } \\
\hline HAD, depression ${ }^{\mathrm{c}}$ & $5.7 \pm 3.4$ & $5.1 \pm 2.6$ & NS \\
\hline $\mathrm{HAD}$, anxiety ${ }^{\mathrm{c}}$ & $6.9 \pm 3.6$ & $6.9 \pm 3.5$ & NS \\
\hline POMS, global score ${ }^{\mathrm{c}}$ & $25.9 \pm 31.6$ & $21.9 \pm 22.1$ & NS \\
\hline POMS, tension-anxiety ${ }^{\mathrm{c}}$ & $7.7 \pm 6.3$ & $6.7 \pm 5.3$ & NS \\
\hline POMS, depression-dejection ${ }^{\mathrm{c}}$ & $12.3 \pm 9.7$ & $10.7 \pm 6.7$ & NS \\
\hline POMS, anger-hostility ${ }^{\mathrm{c}}$ & $8.2 \pm 7.2$ & $6.7 \pm 6.3$ & NS \\
\hline POMS, vigor-activity ${ }^{\mathrm{d}}$ & $-15.2 \pm 4.3$ & $-13.3 \pm 5.5$ & NS \\
\hline POMS, fatigue-inertia ${ }^{c}$ & $8.6 \pm 5.4$ & $7.4 \pm 3.9$ & NS \\
\hline POMS, confusion-bewilderment ${ }^{\mathrm{c}}$ & $4.6 \pm 4.4$ & $3.5 \pm 3.6$ & NS \\
\hline Quality of life, global score $\mathrm{e}^{\mathrm{e}}$ & $81.3 \pm 10.2$ & $79.7 \pm 10.3$ & NS \\
\hline
\end{tabular}

androgens to estrogens in adipose tissue) and mechanical factors (on weight-bearing bone sites), but leptin also probably mediates fat and bone mass [20]. Leptin is an anorexigenic metabolic hormone that is secreted in proportion to fat mass [23] and decreases bone formation [24]. A strong positive correlation between fat mass and serum leptin concentrations has been reported [25].

In our study, results of cognitive functions are worse, risk factors predicting hip fractures are higher, but physical activity is also higher in women with osteoporosis. In addition, we found some correlations between physical activity and emotional state and quality of life scores.

There is convincing evidence that physical activity effectively slows bone loss in postmenopausal women in a dose-dependent manner. An inverse relationship between physical activity and the risk of hip fracture was detected [26]. Physical activity improves balance, co-ordination, muscle strength, and reaction time. All these traits increase the possibility to break a tendency to fall or to protect from the full impact of an actual fall by the arms [27, 28].

In our study, results of cognitive functioning in postmenopausal women with osteoporosis were worse than in age- and sex-matched controls. Other studies show similar results. Women with osteoporosis were found to have poorer cognitive functioning and greater risk to cognitive deterioration [29]. Besides, women with more rapid hipbone loss were more likely to develop cognitive decline than those who had lower rate of loss (or who gained bone mass) [30]. Cognition modified the association between BMI and hip fracture in a large cohort of older persons. Though underweight $(\mathrm{BMI}<22)$ individuals were at greater risk of hip fracture, the risk was greater among persons with lower results of performing cognitive functioning tests. Low cognition has been shown to compromise the adaptive and coping abilities of older adults to environmental demands or medical stressors. The lack of ability to adapt may translate into less physical activity, poor adherence to treatment regimen, and poor motivation to engage in healthy lifestyles. On the other hand, cognitively impaired older adults who maintain good mobility may reduce their risk of fractures [31].

Probably because of too small sample size or because of the quite active way of life of participants, we did not find significant differences in quality of life or emotional state of osteoporotic patients and healthy controls in contrast to data of other studies.

Health-related quality of life, assessed by generic and osteoporosis-specific instruments, is decreased in patients
Table 3 Significant correlations between physical activity and psychological data

\begin{tabular}{llcc}
\hline Correlations between & & $r$ (Pearson test) & $p$ value (Pearson test) \\
\hline Physical activity & HAD, depression & -0.270 & 0.042 \\
Physical activity & HAD, anxiety & -0.301 & 0.023 \\
Physical activity & POMS, depression-dejection & -0.278 & 0.033 \\
Physical activity & POMS, global score & -0.263 & 0.046 \\
Physical activity & Quality of life, global score & 0.269 & 0.041 \\
\hline
\end{tabular}


with vertebral fractures due to osteoporosis as a function of the number of vertebral fractures, presence of comorbid conditions, and age [32]. A strong positive association was found between health-related quality of life and postsecondary education, a family history of osteoporosis, and working. Living in long-term care has the most marked negative effect on quality of life [33]. Although the quality of life does not correlate with bone mineral density, it is markedly influenced by the presence of vertebral fractures, especially recent ones [34, 35].

Women with osteoporosis have the greatest impairments in physical, psychological, and social aspects of quality of life [36].

The long-term physical, psychological, and social consequences affect quality of life, impair social and leisure activities, and may alter emotional status to the extent of causing depression [8].

Osteoporosis, as a chronic illness, influences interpersonal relationships [30]. Since, for many people, the first vertebral fracture occurs without impairment or pain, it usually has no psychosocial effect. When such symptoms as functional disability, pain, and deformity occur, psychosocial functioning diminishes [37].

In the social arena, these include social role loss (roles of the individual and family roles), failure in social reciprocity, and social isolation [38, 39].

The social outcomes often precede other consequential problems, including those in the psychological arena. The two primary psychological issues associated with osteoporosis are anxiety and depression [1].

Anxiety (feelings of apprehension and fear) is a normal emotion that occurs when people face an unknown or threatening situation. Many women experience severe anxiety when they are diagnosed with osteoporosis, especially when an atraumatic, but symptomatic, fracture leads to diagnosis [38]. In the context of osteoporosis, anxiety often leads patients to overexert themselves in making lifestyle changes so that, after a short while, they find themselves overwhelmed and unable to comply with most of the changes [1].

The relationship between osteoporosis and depression is a tantalizing one, yet no longitudinal studies have been conducted that allow researchers to make causal attribution or to really understand the potential interactive mechanisms of the diseases [1]. It was suggested that depression is associated with lower neuroendocrine function and, therefore, lower bone density [40]. In public health terms, both osteoporosis and depression are major problems. Combining them has the potential to create an even worse challenge for individuals [1]. Depressive symptoms were found to be associated with an increased rate of bone loss [41].

Oxidative stress is associated with the risk of osteoporosis [42]. Other risk factors for osteoporosis such as smoking, hypertension, and diabetes mellitus are also associated with increased oxidative stress and free-radical levels [43].

The main limitation of our study was the relatively small sample size. Another limitation was not assaying hormonal and vitamin D levels in the blood of participants. In addition, we did not evaluate socioeconomic state, presence or absence of other chronic diseases, presence or absence of fractures, diet, and the use of supplements. These limitations are also our guidelines for further research works.

Although the study has some limitations, it revealed many topics in the biopsychological and health-related well-being situation of postmenopausal women with osteoporosis in Lithuania. These results could be important for all the health and social support system specialists giving treatment and support for their patients.

Throughout the last decade, researchers have made clear that not all of the consequences of osteoporosis are skeletal in nature. Focusing on the biopsychosocial nature of osteoporosis becomes more critical as the number of people with this diagnosis increases [1].

In conclusion, in postmenopausal women with osteoporosis of Lithuania, weight, BMI, fat body mass, lean body mass, water body mass, basal metabolic rate, and waist-tohip ratio are lower, physical activity and risk of fractures are higher, and cognitive functions are worse than in ageand sex-matched controls. Some psychological peculiarities could be related to physical activity in women with osteoporosis, but it needs further investigation.

\section{Conflicts of interest None.}

Open Access This article is distributed under the terms of the Creative Commons Attribution Noncommercial License which permits any noncommercial use, distribution, and reproduction in any medium, provided the original author(s) and source are credited.

\section{References}

1. Gold DT (2002) Physical and psychosocial consequences of osteoporotic vertebral fracture (Part 2). Identifying and managing the non-skeletal consequences of osteoporosis. Advances in Osteoporotic Fracture Management 1(3):74-79

2. Wallace LS, Ballard JE, Holiday DB, Wells HE (2005) Comparison between 60 matched pairs of postmenopausal black and white women: analysis of risk factors related to bone mineral density. Maturitas 52(3-4):356-363

3. Caliri A, De Filippis L, Bagnato GL, Bagnato GF (2007) Osteoporotic fractures: mortality and quality of life. Panminerva Med 49(1):21-27

4. Lashas L, Masanauskaite D, Lashene D, Masanauskiene E (1996) Rate of characteristic for osteoporotic fractures in the elderly population of Kaunas region, Lithuania. Scand J Rheumatol 25 (Suppl 103):16-20 
5. Alekna V, Tamulaitiene M (2003) Bone mineral density in healthy Lithuanian women (in Lithuanian). Medicina 39(5):498-503

6. Black DM, Steinbuch M, Palermo L et al (2001) An assessment tool for predicting fracture risk in postmenopausal women. Osteoporos Int 12:519-528

7. de la Loge C, Sullivan K, Pinkey R, Roux P, Meunier PJ (2005) Cross-cultural validation and analysis of responsiveness of the QUALIOST: QUAlity of Life questionnaire in Osteoporosis. Health Qual of Life Outcomes 3:69

8. Cohen-Solal ME, de Vernejoul M-C (2002) Quality of life in osteoporosis. Medicographia 24(4):349-352

9. Tolea MI, Black SA, Carter-Pokras OD, Kling MA (2007) Depressive symptoms as a risk factor for osteoporosis and fractures in older Mexican American women. Osteoporos Int 18(3):315-322

10. Silverman SL, Shen W, Minshall ME, Xie S, Moses KH (2007) Prevalence of depressive symptoms in postmenopausal women with low bone mineral density and/or prevalent vertebral fracture: results from the Multiple Outcomes of Raloxifene Evaluation (MORE) study. J Rheumatol 34(1):140-144

11. Reventlow SD (2007) Perceived risk of osteoporosis: restricted physical activities? Qualitative interview study with women in their sixties. Scand J Prim Health Care 25(3):160-165

12. Gold DT, Solimeo S (2006) Osteoporosis and depression: a historical perspective. Curr Osteoporos Rep 4(4):134-139

13. Tashiro A, Kakuta H, Tanaka N, Takeuchi Y (2006) Relationship between health-related quality of life domains and bone status in postmenopausal Japanese women. Menopause 13(5):846-849

14. Alekna V, Tamulaitiene M, Butenaite V (2006) The impact of subclinical vertebral fractures on health-related quality of life in women with osteoporosis (in Lithuanian). Medicina 42(9):744-750

15. McNair O, Lorr M, Droppleman LF (1992) Edits manual: profile of mood state. Educational and Institutional Testing Service, San Diego

16. Zigmond AS, Snaith RP (1983) The hospital anxiety and depression scale. Acta Psychiat Scand 67:361-370

17. Retain RM (1977) A research program of the psychological effects of brain lesions in human beings. In: Ellis NR (ed) International Review of Research in Mental Retardation. England Medical Center, New York

18. Wechsler D (1981) WAIS-R Manual. Psychological Corporation, New York

19. WHO (1996). WHOQOL-BRIEF: introduction, administration, scoring and generic version of the assessment. World Health Organization. Field Trail Version

20. Crepaldi G, Romanato G, Tonin P, Maggi S (2007) Osteoporosis and body composition. J Endocrinol Invest 30(Suppl 6):42-47

21. Hsu Y-H, Venners SA, Terwedow HA, Feng Y, Niu T, Li Z, Laird $\mathrm{N}$, Brain JD, Cummings SR, Bouxsein ML, Rosen CJ, Xu X (2006) Relation of body composition, fat mass and serum lipids to osteoporotic fractures and bone mineral density in Chinese men and women. American J Clin Nutr 83(1):146-154

22. Gnudi S, Sitta E, Fiumi N (2007) Relationship between body composition and bone mineral density in women with and without osteoporosis: relative contribution of lean and fat mass. J Bone Miner Metab 25(5):326-332

23. Auwerx J, Steals B (1998) Leptin. Lancet 351:737-742

24. Takeda S, Elefteriou F, Levasseur R et al (2002) Leptin regulates bone formation via the sympathetic nervous system. Cell 111:305-317

25. Kobyashi J, Sasaki T, Watanabe M (2004) The relationship of abdominal fat mass assessed by helical or conventional computer tomography to serum leptin concentration. J Atheroscler Thromb 11:173-179

26. Schmitt NM, Schmitt J, Döher M (2009) The role of physical activity in the prevention of osteoporosis in postmenopausal women - an update. Maturitas 63(1):34-38

27. Daley MJ, Spinks WL (2000) Exercise, mobility and aging. Sports Med 29:1-12

28. Nelson ME, Fiatarone MA, Morganti CM, Trice I, Greenberg RA, Evans WJ (1994) Effects of high-intensity strength training on multiple risk factors for osteoporotic fractures. A randomized controlled trial. JAMA 272:1909-1914

29. Yaffe K, Browner W, Cauley J, Launer L, Harris T (1999) Association between bone mineral density and cognitive decline in older women. J Am Geriatr Soc 47(10):1176-1182

30. Lui LY, Stone K, Cauley JA, Hillier T, Yaffe K (2003) Bone loss predicts subsequent cognitive decline in older women: the study of osteoporotic fractures. J Am Geriatr Soc 51(1):38-43

31. Alfaro-Acha A, Ostir GV, Markides KS, Ottenbacher KJ (2006) Cognitive status, body mass index and hip fracture in Hispanic older adults. J Am Geriatr Soc 54(8):1251-1255

32. Salaffi F, Cimmino MA, Malavolta N, Carotti M, Di Matteo L, Scendoni P, Grassi W (2007) The burden of prevalent fractures on health-related quality of life in postmenopausal women with osteoporosis: the IMOF study. J Rheumatol 34(7):1551-1560

33. Papaionnou A, Kennedy CC, Ioannidis G, Brown JP, Pathak A, Hanley DA, Josse RG, Sebaldt RJ, Olszynski WP, Tenenhouse A, Murray TM, Petrie A, Goldsmith CH, Adachi JD (2006) Determinants of health-related quality of life in women with vertebral fractures. Osteoporos Int 17(3):355-363

34. Yoh K, Tanaka K, Ishikawa A, Ishibashi T, Uchino Y, Sato Y, Tobinaga M, Hasegawa N, Kamae S, Yoshizawa M (2005) Health-related quality of life (HRQOL) in Japanese osteoporotic patients and its improvement by elcatinin treatment. J Bone Miner Metab 23(2):167-173

35. Brenneman SK, Barrett-Connor E, Sajjan S, Markson LE, Siris ES (2006) Impact of recent fracture on health-related quality of life in postmenopausal women. J Bone Miner Res 21(6):809-816

36. de Oliveira Ferreira N, Arthuso M, da Silva R, Pedro AO, Neto AM, Costa-Paiva L (2009) Quality of life in women with osteoporosis: correlation between QUALEFFO and SF-36. Maturitas 62(1):85-90

37. Haczynski J, Jakimiuk A (2001) Vertebral fractures: a hidden problem of osteoporosis. Med Sci Monit 7:1108-1117

38. Gold DT (2001) The nonskeletal consequences of osteoporotic fractures. Psychological and social outcomes. Rheum Dis Clin North Am 27:255-262

39. Gold DT (1996) The clinical impact of vertebral fractures: quality of life in women with osteoporosis. Bone 18:185S-189S

40. Jahelka B, Dorner T, Terkula R, Quittan M, Bröll H, Erlacher L (2009) Health related quality of life in patients with osteopenia or osteoporosis with and without fractures in a geriatric rehabilitation department. Wien Med Wochenschr 159(9-10):235-240

41. Michelson D, Stratakis C, Hill L et al (1996) Bone mineral density in women with depression. N Eng J Med 335:1176-1181

42. Sheweita SA, Khashhal KJ (2007) Calcium metabolism and oxidative stress in bone fractures: role of antioxidants. Curr Drug Metab 8(5):19-25

43. Cooper C, Gehlbach S (2006) Identification of patients in need of antiosteoporotic treatment: who to treat today? Medicographia 28 (1): $13-20$ 